\title{
Disaster preparedness using risk-assessment methods from earthquake engineering
}

\author{
Maria Battarra ${ }^{1},{ }^{*}$, Burcu Balcik ${ }^{2}$ and Huifu $\mathrm{Xu}^{3}$ \\ (1) University of Bath, School of Management, BA2 7AY, Bath, UK, m.battarra@bath.ac.uk \\ (2) Ozyegin University, Industrial Engineering Department, Nisantepe Mah. Orman Sk., \\ Cekmekoy, 34794 Istanbul, Turkey, burcu.balcik@ozyegin.edu.tr \\ (3) University of Southampton, Mathematical Sciences, SO17 1BJ, Southampton, UK, \\ h.xu@soton.ac.uk \\ *: Corresponding author
}

\begin{abstract}
:
Analyzing the uncertainties associated with disaster occurrences is critical to make effective disaster preparedness plans. In this study, we focus on pre-positioning emergency supplies for earthquake preparedness. We present a new method to compute earthquake likelihood and the number of the affected people. Our approach utilizes forecasting methods from the earthquake engineering literature, and avoids using any probabilistic scenarios. We validate the proposed technique by using historical earthquake data from Turkey, a country under significant earthquake risk. We also present a case study that illustrates the implementation of our method to solve the inventory allocation problem of the Turkish Red Crescent.
\end{abstract}

Keywords: OR in disaster relief, disaster preparedness, earthquake forecasting, inventory allocation 


\section{Introduction}

The frequency, intensity and impact of disasters have increased over the last decades due to a variety of factors including population growth and climate change (European Commission, 2016). The global average annual loss from earthquakes, tsunamis, cyclones, and flooding is 314 billion dollars (United Nations Office for Disaster Risk Reduction, 2015). Moreover, about 19,000 deaths and losses of over 29 billion euros occurred in Europe due to earthquakes alone between 1998 and 2009 (European Geosciences Union, 2017). Logistics account for approximately 80\% of the disaster relief efforts operations (Trunick, 2005; Van Wassenhove, 2006). The importance of applying systematic methods to improve the performance of humanitarian operations has been increasingly highlighted in the Operations Research/Management Science (OR/MS) literature over the past decade. Consequently, the number of studies that focus on improving logistics in different phases of disaster management (such as preparedness, response and recovery) by using OR models and techniques has significantly increased. We refer the interested reader to the review papers by Altay and Green (2006), Van Wassenhove (2006), Caunhye et al. (2012), Leiras et al. (2014), and Özdamar and Ertem (2015).

This paper focuses on preparedness efforts of humanitarian organizations for earthquakes, which occur suddenly and typically create huge demand for emergency supplies. In particular, our study is motivated by the disaster preparedness efforts of the Turkish Red Crescent (TRC), which is the main humanitarian organization in Turkey. Turkey is under significant risk of experiencing severe earthquakes in most of its regions. The most populated areas of the country are close to the North Anatolian Fault, which is one of the most active and longest earthquake faults in the world (Geggel and Writer, 2014). For instance, the earthquake that occurred in Izmit (an industrial city neighboring Istanbul) on the $17^{\text {th }}$ of August, 1999, killed over 17,000 people and left hundreds-of-thousands homeless and in need of aid. Therefore, it is critical for the TRC to have effective earthquake preparedness plans.

As of August 2015, the TRC operates 34 warehouses across the country, where a variety of emergency supplies (such as tents, blankets, kitchen kits) are pre-positioned to improve the local response capacity of the organization. Our communication with the TRC disaster managers reveals that the organization is currently not interested in changing the locations of the warehouses; however, they want to evaluate the effectiveness of the tactical decisions related to the amount of inventory to keep at each warehouse. In the current network, the TRC stocks supplies that would be sufficient to meet shelter and nutrition needs of 50,000 families. The total inventory is allocated among the warehouses in the network primarily based on the population residing in macroareas defined by the 
organization, and considering the capacity of the warehouses. The inventory allocation decisions do not currently consider the earthquake risk which may vary greatly across different regions. In this paper, we present a method to include earthquake risk in making inventory allocation decisions for the TRC.

The pre-positioning of emergency supplies has been widely studied in the humanitarian logistics literature. Previous studies take into account different problem settings (e.g., multiple items, different facility types, network and facility failures), performance measures (e.g., response time, coverage, unsatisfied demand, logistics costs) and modelling approaches (see e.g., Leiras et al. (2014) and Balcik et al. (2016) for survey papers in humanitarian network design and inventory management). In particular, two-stage stochastic programming models are widely used (Grass and Kathrin, 2016); in these models, the first-stage decisions (e.g., locations of warehouses and the inventory to preposition therein) are made prior to the realization of an uncertain event (i.e., the location and size of a disaster), while considering the implications of second-stage decisions (e.g., supply distribution from the warehouses to the demand points), which are made once the uncertainty is resolved (i.e., after the disaster). Moreover, disaster scenarios and their probabilities are usually generated based on historical data, which are typically extracted from databases such as EM-DAT (e.g., Balcik and Beamon (2008); Duran et al. (2011); Rawls and Turnquist (2010)). While this may be a practical approach, there is no unified method to generate these scenarios from the historical data and, even if the same data is used, different disaster scenarios and probabilities can be obtained. Furthermore, the probabilities associated with disaster scenarios are usually very small, and truncating these probabilities to a different degree of accuracy may provide different results.

In this paper, we present a new method to quantify the likelihood of earthquake occurrence and estimate the number of affected people, based on the studies of Campbell (1982) and Samardjieva and Badal (2002), respectively. The demand estimates obtained from applying our method are then incorporated into an inventory allocation model. The proposed method is data-driven and grounded on statistical assessment models from the earthquake engineering literature. While the method is simple, it allows us to represent earthquake risk effectively. There are several studies in the literature that propose disaster preparedness models incorporating the unique aspects of the disasters; e.g., Taskin and Lodree (2011) and Galindo and Batta (2013) consider the dynamic wind speed and intensity information for hurricane preparedness and Garrido et al. (2015) use a flood forecasting method to characterize flood risk. This study will be the first one to utilize risk assessment tools from the earthquake forecasting literature and incorporate them into optimization models to support disaster preparedness. 
In summary, this paper contributes to the literature by:

- introducing forecasting models grounded in the earthquake engineering literature into OR/MS optimization models;

- proposing a data-driven and systematic technique to avoid scenarios and potentially-unstable probability values for estimating demand for relief supplies;

- demonstrating how protected earthquake intensities can be systematically obtained and how they compare to available seismic hazard maps (i.e., maps that show different seismic zones in a region);

- illustrating the calculation and use of protected earthquake intensities and estimated number of affected people within a case study.

Section 2 illustrates our methodology by breaking it down into three steps: Subsection 2.1 presents the method used to forecast the likelihood of an earthquake. Subsection 2.2 demonstrates how the demand of emergency supplies for each region is estimated. Subsection 2.3 introduces the TRC case study and the mathematical model for the allocation of emergency supplies. Section 3 presents computational results in a similar structure; that is, each subsection explains the implementation, validation and analysis for the three main steps of our approach. Finally, Section 4 presents concluding remarks and future research directions.

\section{Methodology}

This paper adapts the earthquake forecasting methods from the earthquake engineering literature to estimate the number of affected people in a region hit by an earthquake. We use these demand estimates in allocating a fixed amount of supplies among the existing warehouses of the TRC. The main steps of the proposed method, which will be explained in detail in this section, are:

1. Earthquake forecasting. For each region that can be potentially hit by an earthquake, estimate the intensity of a representative earthquake (reflecting the earthquake risk in the region based on the intensity and frequency of strong earthquakes in the past, as well as the uncertainty associated with the forecast);

2. Demand estimation. On the basis of the intensity of the representative earthquake and the population density of a region, estimate the expected number of affected people in that region when an earthquake strikes; 
3. Inventory allocation. Allocate the emergency supplies among the warehouses based on the estimated number of affected people determined in the previous step.

The guiding ideas behind the choices made in our modelling approach are:

- The method has to be based on easily accessible data and rely on few parameters. We assume that mathematical models that consider allocating emergency supplies over a large region (such as a country) may not involve a high level of detail (e.g., geodetic information or street-by-street building structures) since such detailed data may not be available or lots of assumptions may need to be made to set essential parameters.

- The method should not be based on geopolitical boundaries. Since the political regions (e.g., counties or provinces) may have heterogeneous characteristics (i.e., size, population density, etc.), basing a risk assessment analysis on these boundaries may be unreliable. For instance, a warehouse may not cover all parts of a large province if that province has an elongated shape. Moreover, the impact of a strong earthquake, which affect multiple provinces and is epicentered in a small province, may not be entirely captured by considering a single region.

- The method adopted from the earthquake engineering literature has to be well-known and validated by the research community; at the same time, it has to be sufficiently simple to be implemented with the available secondary data, and used within an optimization model.

In the subsections below, we provide detailed instructions for each step of our method and justify the rationale of our modelling choices.

\subsection{Likelihood of extreme earthquakes and protected intensity values}

In the earthquake engineering literature, different techniques are used to model the occurrence of earthquake events. The classical and mainstream probabilistic approaches are Poisson-based probability distribution functions, and Markov or Semi-Markov chains. While Poisson models assume that earthquakes occur randomly in time, space and magnitude, Markov models describe subsequent earthquakes as energy release sequences. Markov and Semi-Markov chains capture geophysical characteristics of the earthquake occurrence mechanism, but large amounts of data are required to calibrate the model parameters (Anagnos and Kiremidjian, 1988). The Poisson models are frequently employed since they result in simple mathematical equations that appear to match observations in most case studies (Baker, 2008). A recent performance comparison between the two techniques for Turkey can be found in Ünal et al. (2014). 
The most recent literature in earthquake engineering focused on refining forecasting models and testing their validity; examples include: Kayabali and Akin (2003), Seher and Main (2004), Alvarez (2005), Greenhough and Main (2008), and Davis (2012). These models incorporate sources of information that are either not easily accessible by non-specialists, or are based on more sophisticated statistical models. Therefore, in this study, we base our earthquake forecasting on a simple, datadriven Poisson model; specifically, we use the model by Campbell (1982), who first combined the extreme-value (EV) theory (Gumbel, 1958) with Bayesian probability theory. This model has been used for probabilistic assessment of seismic hazards since 1960s (Benjamin, 1968) and has proved a popular method due to its simplicity, small data requirements (Bayrak and Türker, 2016), and ability to recalculate the risk when new information becomes available (Parvez, 2007). In particular, Campbell (1982)'s method has been used to assess earthquake risk in various seismic zones throughout the world (e.g., California in Campbell (1983), Greece and the adjacent areas in Stavrakakis and Drakopoulos (1995) and Papoulia et al. (2001), South America in Galanis et al. (2002), and the North-East Indian peninsula in Yazdani and Kowsari (2013)). Stavrakakis and Drakopoulos (1995), Galanis et al. (2002) and Yazdani and Kowsari (2013) discuss that the results obtained by using Campbell (1982)'s method are consistent with those obtained by other methods, and Campbell (1982)'s method is successful with assessing seismic risk based on data alone.

\subsubsection{Campbell (1982)'s earthquake forecasting model}

The conventional EV distribution (Epstein and Lomnitz, 1966) models the probability that an earthquake of intensity $m$ (on the Richter scale), greater than $m_{l}$, might happen during a time horizon of length $t$ as:

$$
P\left(M_{\max }>m \mid m_{l}, t\right)=1-e^{-\nu t e^{-\beta\left(m-m_{l}\right)}} .
$$

The value $M_{\max }$ represents the intensity of the strongest earthquake that will happen in the time horizon $t$. Let $m \geq m_{l}$ is an earthquake intensity value, where $m_{l}$ is the threshold intensity by which to judge an earthquake as a disaster. The parameter $\nu$ is the mean rate of occurrence of an earthquake; and $\beta$ is the scale parameter of the earthquake magnitude distribution. Note that $\nu=A / \log 10$ and $\beta=\log _{10} B$, where $A$ and $B$ are the parameters in the well-known Gutenberg-Richter relationship (Richter, 1958):

$$
\log _{10} N=A-B\left(m-m_{l}\right)
$$

In the latter, $N$ is the expected number of earthquakes with magnitude greater than $m_{l}$ per year.

Campbell (1982) improves the EV model by incorporating two sources of uncertainty. Specifically, $\nu$ and $\beta$ are assumed to follow gamma probability distribution functions and they are calibrated 
using Bayesian inference and historical earthquake data. We define $n^{\prime \prime}$ to be the number of historical earthquakes, with strength greater than $m_{l}$, occurred in the time horizon $t^{\prime \prime}$ (the length of time for which historical earthquake data is available). Let $m^{\prime \prime}=\sum_{i=1}^{n^{\prime \prime}}\left(m_{i}-m_{l}\right)$, and $m_{i}>m_{l}$ is the strength of each earthquakes stronger than $m_{l}$ occurred in the time horizon $t^{\prime \prime}$. The density function of the posterior distributions of $\nu$ and $\beta$ are:

$$
\begin{gathered}
f(\nu)=K_{1} \nu^{n^{\prime \prime}-1} e^{-\nu t^{\prime \prime}}, \\
f(\beta)=K_{2} \beta^{n^{\prime \prime}-1} e^{-\beta m^{\prime \prime}},
\end{gathered}
$$

where $K_{1}$ and $K_{2}$ are normalizing constants. Incorporating the Bayesian distributions of earthquake occurrences and magnitudes (2) and (3) into (1) allows for defining a Bayesian distribution of extreme earthquake occurrences.

\subsubsection{Protected intensity values}

One of the innovative contributions of this paper is to make use of the model of Campbell (1982) to obtain a so-called protected intensity value, which incorporates into the model an additional source of uncertainty; the uncertainty in the estimation of $\nu$ and $\beta$ parameters. We adopt a conservative approach and consider a set of likely candidate values for the parameters $\nu$ and $\beta$ (instead of a single numerical value).

Given $m$ and $t$, the function in (1) increases for increasing values of $\nu$ and decreases for increasing values of $\beta$. Therefore, the probability of an earthquake occurring is higher for high values of $\nu$ and low values of $\beta$. Conservative values $\bar{\nu}$ and $\underline{\beta}$ can be, respectively, set as $E_{\nu}\left(1+\%_{D e v}\right)$ and $E_{\beta}\left(1-\%_{D e v}\right)$. For sake of simplicity, we do not use an index to specify the region referring to the parameters $\bar{\nu}$ and $\underline{\beta}$, but $\bar{\nu}$ and $\underline{\beta}$ are region-specific and computed using historical data for the region in question. By imposing in Equation (1) the conservative estimates $\bar{\nu}$ and $\underline{\beta}$, we obtain:

$$
P\left(M_{\max }>m \mid m_{l}, t\right)=1-e^{-\bar{\nu} t e^{-\underline{\beta}\left(m-m_{l}\right)}}
$$

This conditional probability distribution takes into account the errors in estimating $\nu$ and $\beta$, as well as the historical data about the region. This function can be used to design scenarios for stochastic models as was done by Balcik and Beamon (2008), Duran et al. (2011), and Rawls and Turnquist (2010). However, the probability values associated to the occurrence of an earthquake in each scenario would produce very small numbers, which may lead to stability issues within mathematical models. Moreover, the probability values would not be usable in conjunction with demand 
estimation models from the earthquake engineering literature (see Section 2.2).

In our proposed approach, a magnitude value represented by the $(1-\alpha)$-percentile of the conservative distribution (5) is determined by setting:

$$
1-e^{-\bar{\nu} t e^{-\underline{\beta}\left(m-m_{l}\right)}}=1-\alpha
$$

We denote this value as $M_{\alpha, \bar{\nu}, \underline{\beta}} . M_{\alpha, \bar{\nu}, \underline{\beta}}$ is the protected earthquake intensity for a region, which is the reference intensity value associated with the region for the coming $t$ years, assuming that the maximum error on the estimate of $\nu$ and $\beta$ is $\%_{D e v}$, and the probability of earthquake occurrence is $1-\alpha$. We can therefore assign a protected intensity value to each region, representing how risky the region is based on the number and intensity of the past earthquakes.

\subsection{Demand estimation}

The expected number of injured people and casualties in the aftermath of an earthquake depend on a variety of factors, including the characteristics of the earthquake (i.e., shaking intensity, depth) and the characteristics of the area hit by the disaster (i.e., population density, building resilience, landscape). In the literature, multiple papers examine how these factors affect the number of casualties and injured (e.g., Jaiswal et al. (2009); So and Spence (2013); Erdik et al. (2014)). These papers take many relevant factors into account; however, detailed information (e.g., the characteristics of each building in each street) are necessary to use these models. Instead of attempting to apply sophisticated techniques, the practical and simplified model by Samardjieva and Badal (2002) is chosen to estimate the number of casualties and injured people. This paper provides empirical functions to compute the number of injured people or casualties as follows:

$$
\begin{gathered}
\log \left(N_{c}\right)=a(P)+b(P) m \\
\log \left(N_{i} / N_{c}\right)=-0.99+0.21 m
\end{gathered}
$$

where $N_{c}$ and $N_{i}$ denote the number of casualties and the number of injured people, respectively. The coefficients $a$ and $b$ are regression parameters depending on the average population density $P$ of the affected area, and $m$ denotes the intensity of the earthquake. Samardjieva and Badal (2002) provide tabular values of $a$ and $b$ for different values of $P$. Note that the model of Samardjieva and Badal (2002) requires the value $m$ as input. In this study, we use protected intensity values, so that the estimate of casualties and injured people not only reflects population density, but also the risk associated to a region. 
The concept of attenuation of earthquake intensities is also important while estimating the affected population. When an earthquake happens in a region, it is not realistic to assume that areas far from the earthquake epicenter are affected by the same intensity than those areas very close to the epicenter. Christoskov et al. (1990) suggest using attenuation coefficients to reflect the fact that the earthquake intensity (and corresponding demand) decreases further away from the earthquake epicenter. For the Turkish case study, the ad hoc empirical attenuation coefficients in Yarar et al. (1984), which are specifically validated for this country, are taken into account. Section 3.1 describes how the Turkish population residing in a region has been allocated to areas with different perceived intensity values.

\subsection{Inventory allocation model}

In this section, we illustrate using the demand estimates generated by the proposed methodology in an earthquake preparedness model, which particularly focuses on inventory allocation decisions of the TRC.

Figure 1 depicts the location of the TRC warehouses; specifically, each crescent sign on the map represents a warehouse. The organization has determined a total (nationwide) stock level for each item and a target amount of supplies for each macroarea (macroareas are depicted in Figure 1). A macroarea contains multiple provinces, and the target of supplies in a macroarea is determined based on its population. There is not a unified TRC policy for allocating the emergency supplies among the facilities within a macroarea. In case of an emergency, the objective of the TRC is to be able to start distributing emergency supplies in the affected region within two hours (we will assume two hours to be the target time for the transportation of emergency supplies). While this target seems ambitious, the TRC managers explain that it is used as an internal motivational slogan and it is not an official performance target. The TRC groups emergency supplies in kits. Each kit satisfies the needs of a family of five people. Hereafter, we assume the allocation of a single type of emergency supply (i.e., a kit).

In this study, we use a coverage based objective. More specifically, we aim for an equitable allocation of the available supplies among the located warehouses by maximizing the minimum coverage level achieved across potential demand locations, where coverage level is measured in terms of the fraction of the emergency supply demand that can be satisfied within the desired response time (similar to Balcik and Beamon (2008)). This objective particularly focuses on the most critical regions with large expected demands and ensures that these regions are covered as much as possible if a disaster occurs. Similar max-min type objectives are used frequently in designing humanitarian relief networks to ensure equity among aid recipients; for instance, Gormez et al. (2011) minimizes 
the maximum distance between the located facilities and potential disaster regions and Mohammadi et al. (2016) minimizes the maximum difference in demand satisfaction ratios among demand locations while pre-positioning supplies. Dividing scarce supplies among recipients proportional to their demand amounts has also been widely used in humanitarian resource allocation problems (e.g., see Balcik et al. (2008); McCoy and Lee (2014); Noyan et al. (2015)). More recently, deprivation costs, which associate an economic value for human suffering due to lack of access to relief supplies, have been used as a criterion in resource allocation (Perez-Rodriguez and Holguin-Veras, 2015). While there may be different alternatives in modeling equity in resource-limited settings, we use a max-min type objective as it allows us to characterize the effects of using the proposed risk assessment method in a practical way (i.e., by specifying the least covered regions) and show the TRC the benefits of including earthquake risk in making allocation decisions.

Figure 1: Location of TRC warehouses (Source: TRC (August 2015)).

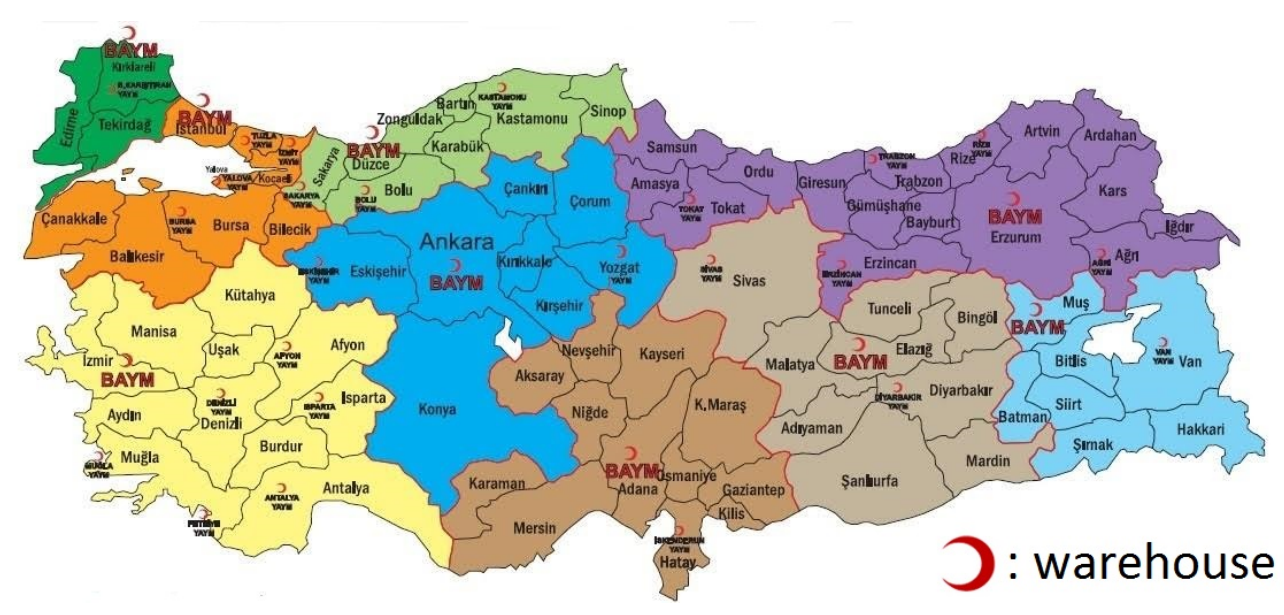

The notation used in our model is as follows. The set of warehouses is represented by $\mathcal{J}$. Each warehouse $j \in \mathcal{J}$ is associated with a capacity. Specifically, warehouse $j \in \mathcal{J}$ can store at most $C_{j}$ emergency kits. The total number of emergency kits to store all over the country is $B$. To model the max-min coverage objective function, we estimate how many emergency supplies would reach each region $i \in \mathcal{I}$ within roughly two hours. We define a coverage coefficient $a_{i j}$, which measures the accessibility of region $i$ from warehouse $j$. We approximate network distances with aerial distances given that the location of the earthquake and the affected areas are not known exactly. The coverage of a region affected by an earthquake is computed as the ratio of the overall amount of supply that can reach the region (weighted by the $a_{i j}$ coefficients) and the expected demand of supplies for the region. 
In our model, variable $x_{j}$ represents the amount of emergency supplies stocked in warehouse $j \in \mathcal{J}$ and variable $z \geq 0$ is the coverage level of the least covered region. The model can therefore be formulated as follows:

$$
\begin{aligned}
& \max z \\
& \text { s.t. } x_{j} \leq C_{j}, \quad \forall j \in \mathcal{J} \\
& \sum_{j \in \mathcal{J}} x_{j} \leq B \\
& \frac{\sum_{j \in \mathcal{J}} a_{i j} x_{j}}{D_{i}} \geq z . \quad \forall i \in \mathcal{I}
\end{aligned}
$$

The objective function (8) maximizes the minimum coverage level. Constraints (9) ensure that the capacity of each warehouse is respected. Constraints (10) restrict the overall number of emergency kits in the country. Constraints (11) define the minimum coverage level among all regions. Note that the coverage level of each region $i \in \mathcal{I}$ is computed as the ratio between the quantity of emergency supplies that can reach region $i$ and the expected demand of emergency supplies $D_{i}$. We allow variables $x_{j}$ to be continuous. This assumption is due to the fact that the emergency kits are available in large numbers and rounding their values to the closest integer would not significantly affect solution accuracy. This allows the TRC to use publicly available software (i.e., Excel solver) to solve the model in negligible time. Note also that the data required by the model is already available to the organization and no significant assumptions are needed to implement it.

The proposed model does not involve additional complexities such as recent pre-positioning models in the literature (such as the post-disaster conditions of facilities and of the transportation network). We propose a simple model, which allows us to test the behaviour of the protected earthquake intensity $M_{\alpha, \bar{\nu}, \underline{\beta}}$, without distortions due to extra constraints and objectives (additional features can be easily incorporated in this the model once the $D_{i}$ values are estimated).

\section{Implementation, results and analysis: TRC case study}

In this section, we describe the implementation of the proposed method on the TRC network and present results. Subsections 3.1 and 3.2 explain how we obtain protected intensity values and estimated demand for the Turkish case, respectively. Subsection 3.3 discusses the results obtained by the inventory allocation model. 


\subsection{Calculating and validating protected earthquake intensity}

In this subsection, we describe the application of the earthquake forecasting method to Turkey in detail.

\subsubsection{Processing the historical earthquake data.}

We first divide Turkey into grids, where each rectangle identified by the grid represents a region potentially affected by an earthquake. We consider the extreme coordinates of Turkey (WIKI, 2015) and draw a large rectangle that contains the entire country. This rectangle is divided into smaller rectangles by splitting the country's longitude and latitude into six equally long segments (see the upper left map in Figure 2, where circles denote the current warehouses' locations). Each of the 36 small rectangles is roughly 270 kilometers $(\mathrm{km})$ in length and $120 \mathrm{~km}$ in width. The rectangles are sized so as to enclose the most disastrous effects of a large earthquake. This grid size proves large enough to capture a sufficient number of historical earthquakes (so that the statistical analysis described in Section 2.1 is relevant). As reported later in Figure 3, 97\% of the regions had more than 5 earthquakes, and $81 \%$ more than 10 earthquakes. Moreover, this grid size allows for capturing the effects of a typical strong earthquake in Turkey (Table 3).

Considering 36 rectangles may not be sufficient to capture the effects of the earthquakes happening on the borders between rectangles. The city of Istanbul, for example, is situated roughly at the intersection of four rectangles (see the upper left map in Figure 2). The effects of an earthquake happening in the city would not be properly captured if we assume earthquakes are confined to a single rectangle. We therefore include in our analysis a larger number of rectangles, so that each border is, in turn, the center of a rectangle. More precisely, the rectangles are shifted horizontally half of their width to obtain the 30 rectangles in the upper right map in Figure 2, they are then shifted vertically up half of their height to obtain further 30 rectangles (bottom left map in the figure), and finally both shifts are applied to obtain the 25 rectangles in the bottom right map. The overall number of rectangles falling completely within the extreme coordinates of Turkey is 121 . Each disaster is assumed to affect a single rectangle (region) at a time, and one rectangle encloses the effects of a disaster. The supplies can be deployed by the warehouses that can cover the rectangle.

We collect data on the past earthquakes that occurred between 1905 and 2015 from the Bogazici University Kandilli Observatory and Earthquake Research Institute (KOERI), which records all ground movements in the country (KOERI, 2015). This database is consulted for the earthquakes with an intensity greater than 5 , depth smaller than $60 \mathrm{~km}$, which occurred in the large rectangle bounding the country. These values are selected as earthquakes of lower intensity and larger depth 
Figure 2: Regions considered in Turkey.
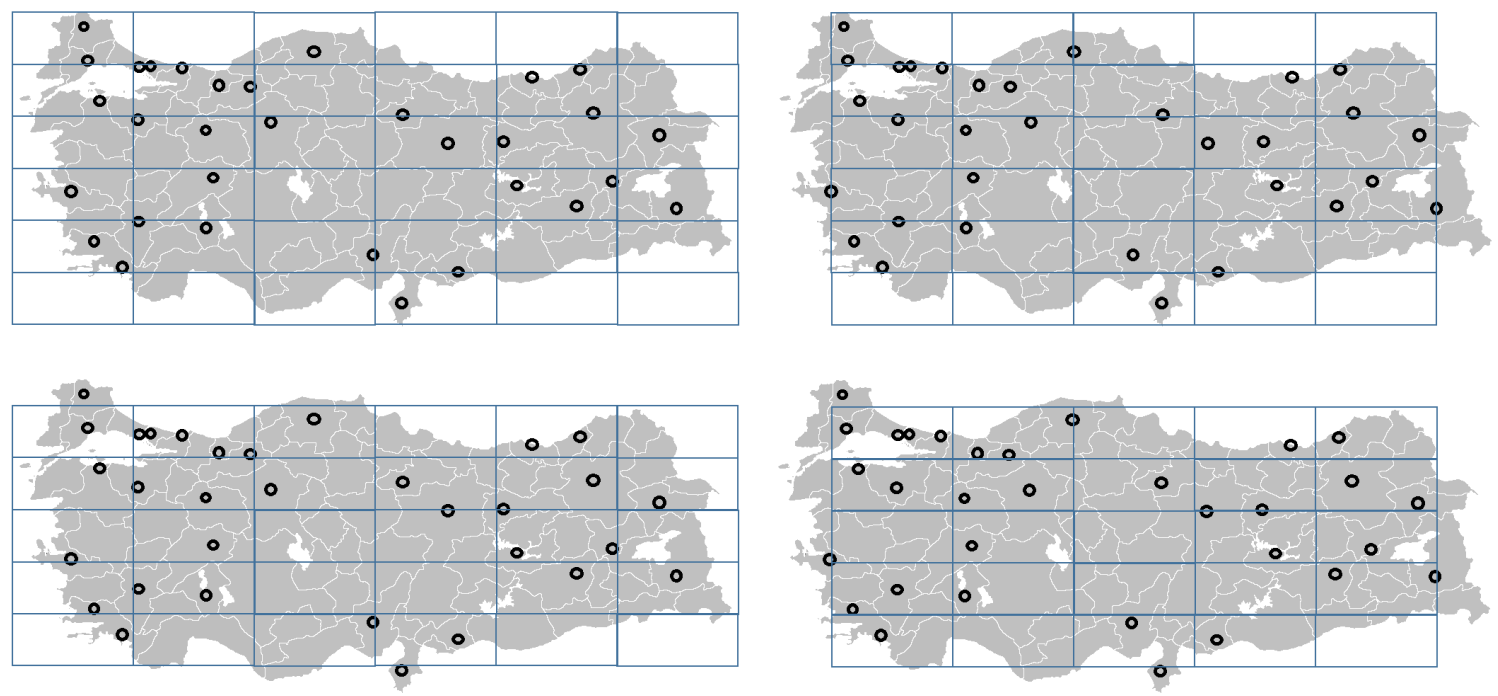

do not typically require large amounts of emergency supplies, as suggested by the related literature discussed in Section 2.2. The data set reports 1087 events.

For each of the 121 rectangular regions, we record the earthquakes falling within the borders of each region (the same earthquake can be associated with multiple rectangles). Then we compute the protected earthquake intensities for each region. We consider a time horizon $t=5$ years for all regions, and set $m_{l}=5$ (as suggested by Samardjieva and Badal (2002)).

\subsubsection{Setting the parameter values.}

We perform tests to set reasonable values for the $\alpha$ and $\%$ Dev parameters. As a rule of thumb, $\alpha$ should be close to 1 , given that the probability of occurrence of an earthquake with magnitude greater than $m_{l}=5$ in the coming five years is expected to be a small number (in particular for low risk regions). We therefore test the values of $\alpha$ between 0.95 and 0.99 . We set $\%_{\text {Dev }}$ as $5 \%$ during these tests. The distribution of the resultant protected earthquake intensities is summarized in Table 1, in which the maximum and average $M_{\alpha, \bar{\nu}, \underline{\beta}}$ values are reported for the 121 regions of Turkey. Based on our analysis, we set $\alpha$ at 0.98 since the strongest earthquake ever reported by the KOERI Observatory has an intensity of 7.9 , and we choose to maintain the maximum value of the protected earthquake intensity to be below 8.0.

The value of $\%_{D e v}$ is an estimate of the error expected in forecasting the number and intensity of earthquakes of strength over 5.0 for each region across the country. A planner has to carefully calibrate this parameter. A large value of $\%$ Dev might overprotect regions with few historical earth- 
Table 1: Protected earthquake intensities with respect to varying values of $\alpha$

\begin{tabular}{l|ccccc}
$\alpha$ & 0.95 & 0.96 & 0.97 & 0.98 & 0.99 \\
\hline Avg. $M_{\alpha, \bar{\nu}, \underline{\beta}}$ & 6.186 & 6.292 & 6.429 & 6.621 & 6.948 \\
Max. $M_{\alpha, \bar{\nu}, \underline{\beta}}$ & 7.368 & 7.519 & 7.712 & 7.984 & 8.501
\end{tabular}

Table 2: Protected earthquake intensity levels with respect to varying values of $\%_{\text {Dev }}$

\begin{tabular}{l|ccccc}
$\%_{\text {Dev }}$ & $25 \%$ & $10 \%$ & $7.5 \%$ & $5 \%$ & $2.5 \%$ \\
\hline Avg. $M_{\alpha, \bar{\nu}, \underline{\beta}}$ & 6.983 & 6.694 & 6.657 & 6.621 & 6.587 \\
Max. $M_{\alpha, \bar{\nu}, \underline{\beta}}$ & 8.549 & 8.057 & 8.020 & 7.984 & 7.948 \\
\hline$M_{\alpha, \bar{\nu}, \underline{\beta} \text { Ala }}^{\text {Alanbey }}$ & 7.346 & 6.579 & 6.487 & 6.401 & 6.320 \\
$M_{\alpha, \bar{\nu}, \underline{\beta}}^{\text {Dursunbey }}$ & 7.176 & 7.056 & 7.039 & 7.022 & 7.055
\end{tabular}

quakes, a small value might return protected intensities even below $m_{l}$ (this happened with other data sets and will be explained in the Appendix). In Table 2, we summarise how the parameter \% Dev was set for this case study (having fixed $\alpha=0.98$ ).

Table 2 shows the average and maximum protected earthquake intensity levels across all regions of the country (first row) and for specific regions with low and high earthquake occurrences in the past. Alanya is a Southern city where only three strong earthquakes have been recorded since 1905, with average intensity 5.63. Dursunbey is a town on the West coast, located roughly half way between Istanbul and Izmir, that suffered 81 earthquakes of average intensity 5.38 in the same time horizon. The region North of Dursunbey, which covers Istanbul, the Marmara Sea, and the South coast of the Marmara Sea, have the highest risk, according to our method. The values of intensity for this high-risk region are reported in the second row (denoted by Max. $M_{\alpha, \bar{\nu}, \underline{\beta}}$ ) in Table 2. This region, which is closest to the North Anatolian fault, suffered 42 earthquakes with an average intensity 5.643 and our model estimates the protected intensity as 7.984 (with $\%_{\text {Dev }}=5 \%$ ).

Regions like Alanya should not be overprotected. Note that a large $\%_{D e v}$ value (i.e., $25 \%$ ) brings the $M_{\alpha, \bar{\nu}, \underline{\beta}}^{\text {Alanya }}$ to an intensity value higher than that of Dursubey. We set $\%_{\text {Dev }}=5 \%$, since this value maintains the strength of the maximum protected intensity below 8.0, and, at the same time, Alanya is not considered riskier than, for example, Dursunbey.

In setting our parameters for Turkey, we also compared protected intensity values with the corresponding expected intensities (the intensities obtained using the expected $\nu$ and $\beta$ from (6) and (7)). The difference is at most an increase of 0.23 on the Richter scale with respect to the corresponding expected intensity (for regions with few strong earthquakes). However, the use of protected intensi- 
ties becomes very important when datasets are sparse and regions have heterogeneous characteristics (i.e., some regions are affected by very strong earthquakes, while others have no record of strong earthquakes in the past). The Appendix illustrates the importance of including the $\%$ Dev parameter when considering historical data for China. More specifically, the Chinese case shows that one can obtain protected intensity values within the desired range even for regions with very different risk profiles by calibrating the $\%$ Dev parameter.

\subsubsection{Testing the effect of the grid size.}

We used a sensitivity analysis to assess the robustness of our choice of the size of the regions. We counted the number of earthquakes occurring in each region by dividing the country into $4 \times 4$, $5 \times 5,6 \times 6$, and $7 \times 7$ rectangles. We also counted the regions where the protected intensity values represented no risk (equal to 5.0 intensity), very low risk (between 5.0 and 5.5), low risk (between 5.5 and 6.0 ) and so on by varying the grid size. Our results are depicted in Figure 3 . A $4 \times 4$ grid would result in $66 \%$ of the territory being high risk (over 6.5 protected intensity), with some areas having a protected intensity of over 8.0 (which has never occurred according to the historical data). Conversely, an $8 \times 8$ grid would lead to $54 \%$ of the territory being low risk (below 6.5 earthquake intensity) and only $13 \%$ of the territory being high risk (over 7.5 intensity). The $6 \times 6$ grid solution presents protected intensities well spread across the range of risk. Additionally, the solutions with $5 \times 5$ or $7 \times 7$ grids, would still provide sufficiently well distributed results.

Figure 3: Number of earthquakes and protected intensity values varying with the grid size

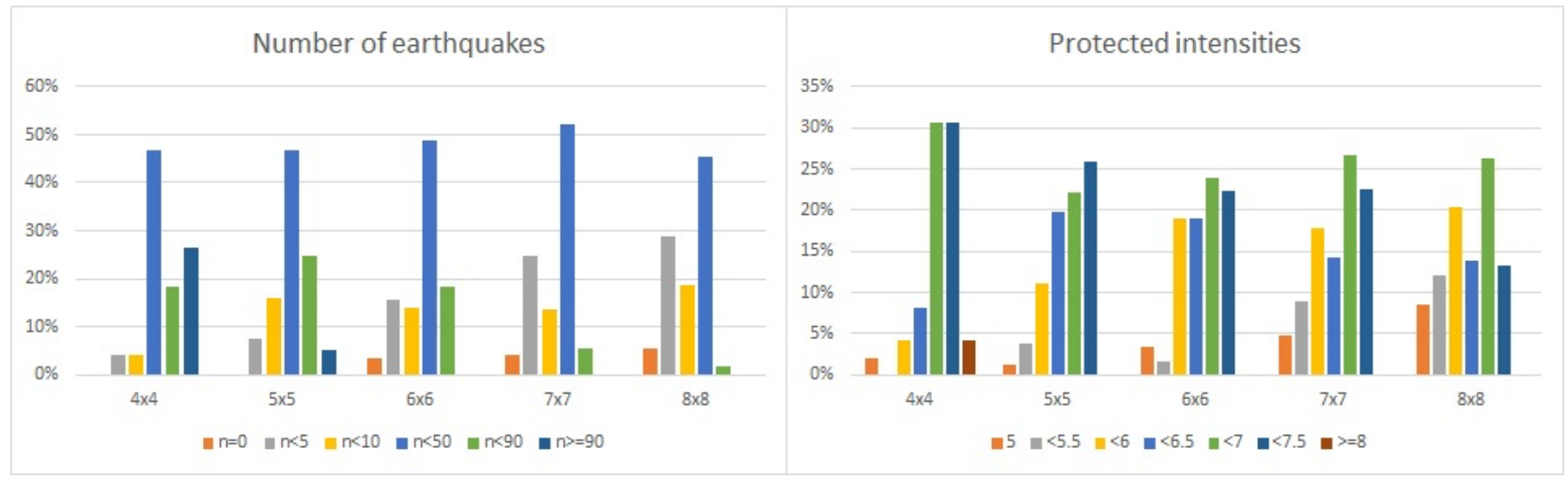

\subsubsection{Validation of the protected intensity values.}

We validate the values of $M_{\alpha, \bar{\nu}, \underline{\beta}}$ by comparing them with publicly available hazard maps, in particular, the one compiled under the Global Seismic Hazard Assessment Program (GSHAP, 2015). 
Hazard maps are practical tools showing how earthquake hazards vary across a given region. The colors in the maps typically reflect the maximum horizontal ground acceleration expected in each region, computed using either probabilistic approaches (e.g., Erdik et al. (1985)) or deterministic approaches (e.g., Kayabali and Akin (2003)), by considering the geological and topographical characteristics of the ground.

We map the $M_{\alpha, \bar{\nu}, \underline{\beta}}$ values onto linear scales of hazard, and associate a color with each of them, similarly to the hazard map in analysis; specifically, regions with protected intensity values between 5.5 and 6.0 are depicted in light cream, between 6.0 and 6.5 in yellow, 6.5 and 7.0 in pink, and over 7.0 in red. The results are summarized in Figure 4. Despite its simplified nature, our technique is capable of identifying the areas of Turkey most at risk and provides a good match to the available hazard map.

Figure 4: Protected intensities and hazard maps, Turkey
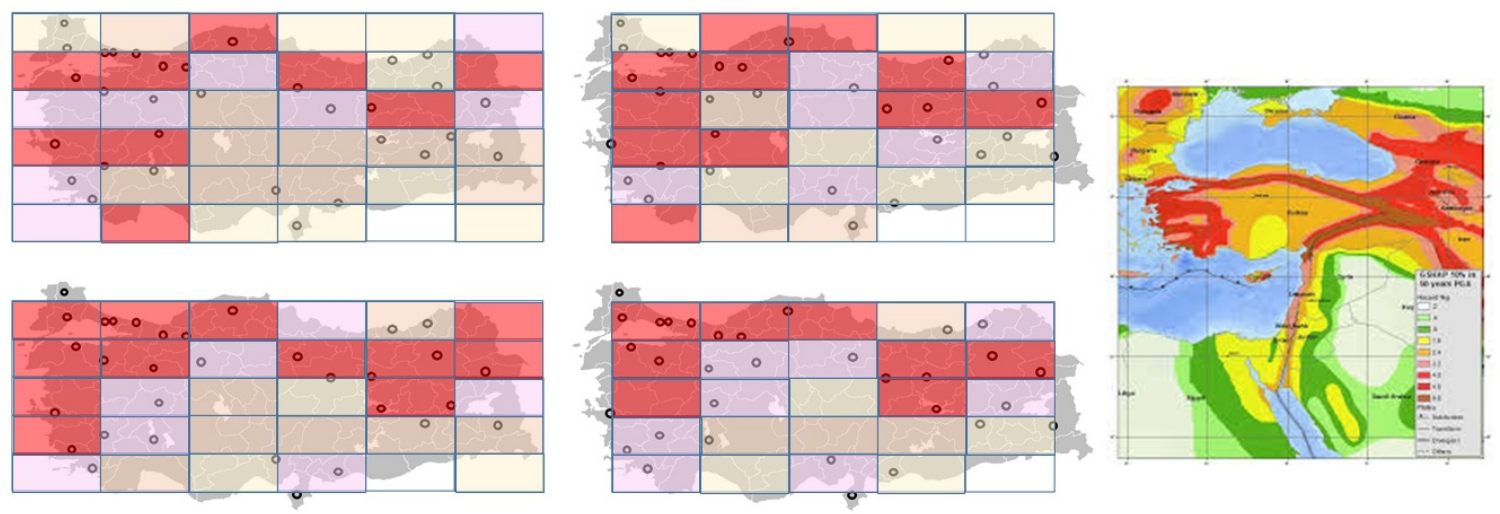

\subsection{Estimation of the number of affected people}

The intensity of an earthquake decreases as the further one gets from the epicenter. Yarar et al. (1984) depict the perceived strength of an earthquake in Turkey as a function of the distance from the epicenter. For example, if a region is hit by an earthquake of strength $7.0 \leq M_{\alpha, \bar{\nu}, \underline{\beta}} \leq 8.0$, Yarar et al. (1984) provide the radius $r$ of the circular area (rooted in the epicenter) in which the earthquake is perceived at strength $M_{p}=7$, as well as the radius of the circular area up to which the strength of the earthquake is perceived at intensity over $M_{p}=6$, and finally, the radius of the area with perceived intensity over $M_{p}=5$ (assuming Graben type earthquakes).

Table 3 considers earthquakes of protected intensities $M_{\alpha, \bar{\nu}, \underline{\beta}}$ over $8,7,6$, and 5 (at the epicenter). The radii $r$ of the areas in which the earthquake perceived intensity $M_{p}$ is over $8,7,6$, and 5 , are given. 
Table 3: Areas affected by an earthquake

\begin{tabular}{l|llll}
$M_{\alpha, \bar{\nu}, \underline{\beta}}$ & $M_{p}$ & $r$ & $A_{F}$ & $A_{E}$ \\
\hline$\geq 8$ & 8 & 50 & $7,853.98$ & $7,853.98$ \\
& 7 & 100 & $31,415.93$ & $23,561.94$ \\
& 6 & 200 & $125,663.71$ & $94,247.78$ \\
\hline$\geq 7$ & 7 & 30 & $2,827.43$ & $2,827.43$ \\
& 6 & 70 & $15,393.80$ & $12,566.37$ \\
& 5 & 150 & $70,685.83$ & $55,292.03$ \\
\hline$\geq 6$ & 6 & 20 & $1,256.64$ & $1,256.64$ \\
& 5 & 45 & $6,361.73$ & $5,105.09$ \\
\hline$\geq 5$ & 5 & 15 & 706.86 & 706.86
\end{tabular}

$A_{F}$ is the area in $\mathrm{km}^{2}$ of the circle with radius $r$, and $A_{E}=\pi\left(r^{2}-r_{i n t}^{2}\right)$ is a ring-shaped area defined by the outer radius $r$ and inner radius $r_{i n t}$ (the radius of the largest circular area corresponding to a stronger $M_{p}$, if any).

In this study, we extract population density data from SEDAC (2015), which provides estimations of the population residing in 2.5 arc-minute grid cells for the whole world. We assume that the population in a $5 \times 5$ degree area reside in a region of Turkey (regions are the rectangles defined in Section 3.1), if the center of the given area falls within the coordinates of the region. We then adopt a conservative approach to allocate the population in a region to the areas affected by different earthquake intensities. We assume that the most crowded areas are affected by the earthquake at the strongest intensity. For example, for an earthquake of an intensity between 7.0 and 8.0, we assign the most densely populated areas in the region for up to $2827.43 \mathrm{~km}^{2}$ to be the closest to the epicenter (see Table 3 ). Then we allocate the remaining most densely populated areas to a perceived earthquake intensity 6.0 (up to covering $12566.37 \mathrm{~km}^{2}$ ), and finally, the remaining population in the region is assumed to be affected by earthquake intensity 5.0. For each perceived earthquake intensity area, the average population density $P$ value is computed, and finally, the number of affected people is calculated by using Formulas (6) and (7). A graphical representation of the number of affected for each region is depicted in Figure 5; here different colors depict regions with a higher or a lower number of estimated affected people.

\subsection{The inventory allocation model: analysis and results}

In this section, we discuss the solutions obtained by our model and present the results of the tests performed on critical model parameters. The mathematical model was coded in C using the CPLEX Callable Library, and CPLEX 12.6 was used as the optimization solver. The negligible computing times (i.e., less than a second) are not reported. 
Figure 5: Estimated casualties and injured people, Turkey.

\begin{tabular}{|c|c|c|c|c|c|}
\hline$\circ$ & & $\circ$ & L & & 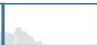 \\
\hline 0 & 0 & & & 00 & \\
\hline & $\circ$ & $\circ$ & 。 & 6 & $\circ$ \\
\hline 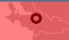 & - & 7 & & $\begin{array}{ll}0 & 0\end{array}$ & 0 \\
\hline 0 & 0 & & $d$ & F & 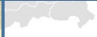 \\
\hline & 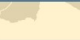 & ק & 0 & & \\
\hline
\end{tabular}
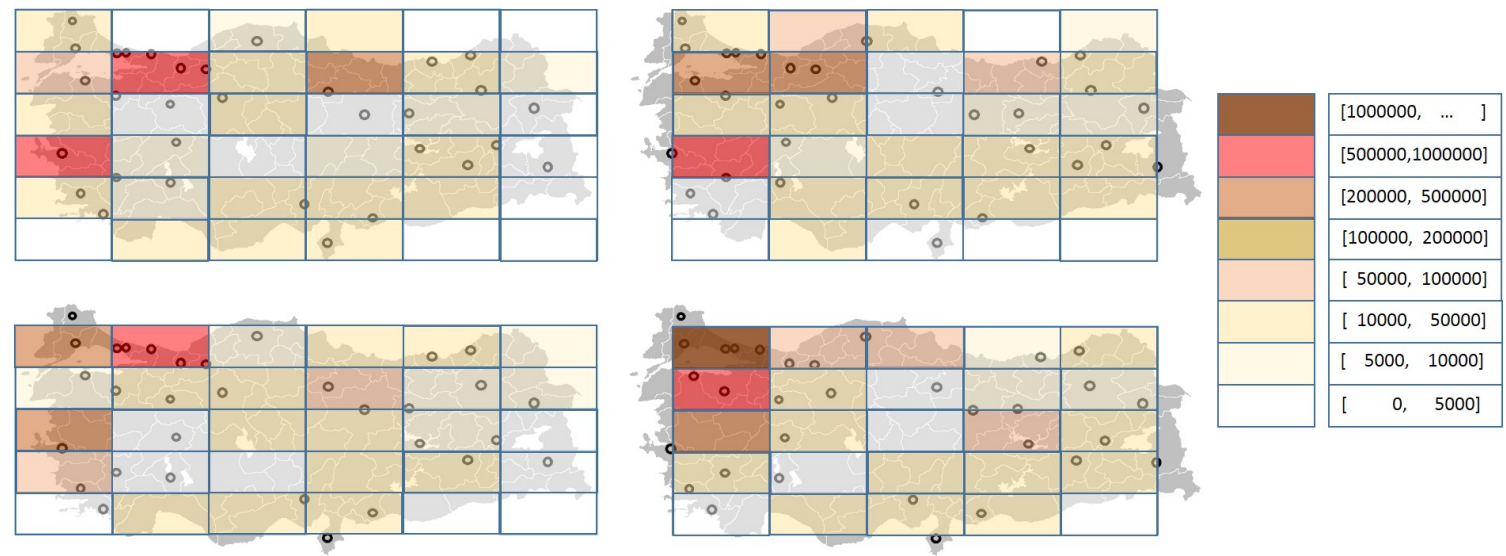

We set the model parameters as follows. Given region $i$ and warehouse $j$, the value of the coverage parameter $a_{i j}$ is set to 1 if the aerial distance from $i$ and $j$ is below $100 \mathrm{~km}, 0.5$ if the aerial distance is between $100 \mathrm{~km}$ and $150 \mathrm{~km}, 0.25$ if the aerial distance is between $150 \mathrm{~km}$ and $200 \mathrm{~km}$, and 0.1 if the aerial distance is between $200 \mathrm{~km}$ and $300 \mathrm{~km}$. These values are set by considering the 2-hour response time target of the TRC. Furthermore, the number of kits available nationwide is $B=50,000$. The $C_{j}$ parameters are set based on the actual warehouse capacity data obtained from the TRC.

We first compare the coverage values obtained by our approach and the TRC's current allocation policy. In calculating coverage levels for both cases, we set the expected demand $\left(D_{i}\right)$ values as explained in Section 3.2. The TRC currently allocates inventory in its network in proportion to the population residing in each macroarea, and furthermore does not utilize an optimization model to determine what inventory to keep within each macroarea. We calculate the minimum coverage levels based on the stock levels at the TRC warehouses at the time the data was received (August 2015). We refer to this solution as the current network or current solution. We find that the minimum coverage percentage in the current network is $1.93 \%$. We note that 49,561 supply kits were stocked in the current network, which is slightly smaller than the overall capacity (i.e., $B=50,000$ ).

The TRC has also provided us with the target stocks (i.e., the maximum number of emergency kits that the TRC aims to store) for each macroarea. The inventory allocation model is executed by imposing and then not imposing the macroarea constraints. The macroarea constraints are formulated as follows:

$$
\sum_{j \in \mathcal{M}_{k}} x_{j} \leq T_{k}, \forall k \in \mathcal{M}
$$


Table 4: Minimum coverage levels and number of warehouses used for different number of kits

\begin{tabular}{l|l|cc} 
& $\begin{array}{l}\text { Number of } \\
\text { kits }\end{array}$ & $\begin{array}{c}\mathrm{Z} \\
(\%)\end{array}$ & $\begin{array}{c}\text { \# warehouses used } \\
\text { (out of 34) }\end{array}$ \\
\hline Current solution & 49,561 & 1.93 & 32 \\
\hline \multirow{3}{*}{ Proposed solution } & 50,000 (Constraints (12)) & 1.72 & 14 \\
& 50,000 & 9.52 & 17 \\
& 55,000 & 10.53 & 16 \\
& 60,000 & 11.49 & 16 \\
& 100,000 & 19.04 & 17
\end{tabular}

where $\mathcal{M}_{k}$ is the set of warehouses in the $k$-th macroarea, $T_{k}$ the maximum number of emergency kits in macroarea $k$ and $\mathcal{M}$ the set of all the macroareas.

The minimum coverage percentage found by this model is $1.72 \%$; that is, the coverage levels obtained by using our allocation model with macroarea constraints and the current solution are similar. However, if Constraints (12) are relaxed, the optimal minimum coverage percentage obtained by our model becomes $9.52 \%$, which is a significant increase from the current coverage value (Table 4 ). This suggests that the macroareas as currently set by the organization are restrictive. Therefore, we do not consider these constraints in further computational analysis and refer to the solution without Constraints (12) as the proposed solution. Additional tests, which assume a larger capacity at each facility (and the same budget $B$ ), do not provide a significant improvement to the coverage. Then, we examine the effects of increasing the number of emergency kits nationwide by increasing the budget $B$ gradually; in these tests, we set $C_{j} \times 2.0$ as for the capacity of each warehouse (allowing for some slack from the current stock levels with a larger budget). The results are summarised in Table 4. By doubling the number of emergency supplies, the minimum coverage level reaches $19.04 \%$. From the results, we also observe that the warehouses in Bursa and Tuzla (both in the south of Istanbul) are typically filled up to capacity, even in scenarios with large $B$ values. This highlights the strategic importance of these warehouses for the TRC.

Figure 6 depicts the regions with coverage below $9.52 \%$ in the current solution. The proposed model is therefore providing better coverage for areas in which both the population and the risk are high. Note, for example, the regions in the North coast of Turkey, which receive few supplies in the current network, as well as the region in the South of Turkey (near Antalya).

Figure 7 provides a visual of the variations in coverage from the current to the proposed solution. For ease of representation, we depict the first 36 regions only and do not report the coverage variations for those regions in which there is no population residing (i.e., they fall in the sea) or the coverage is more than $100 \%$ both before and after using optimization. The two numerical values written within 
Figure 6: Regions with improved coverage in the proposed solution

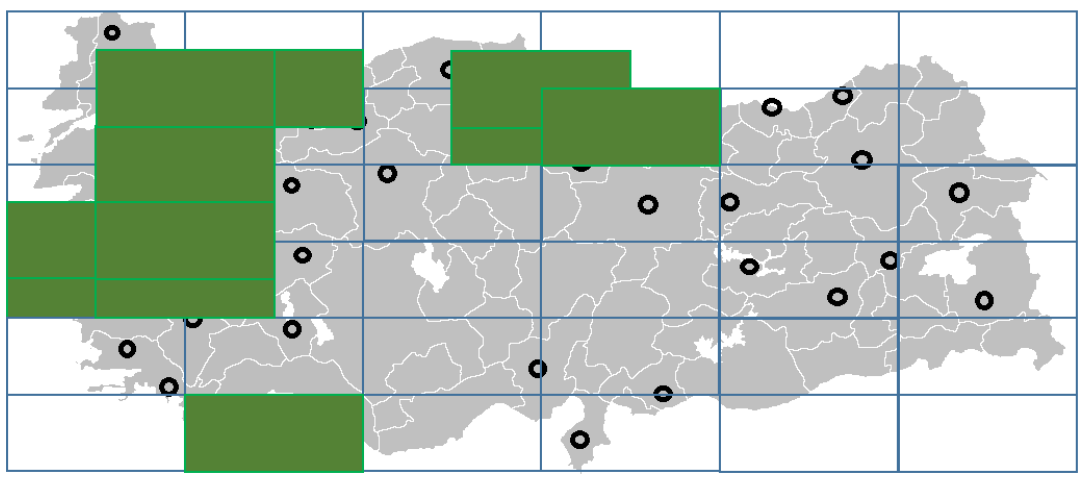

each region boundaries are the coverage in the current solution (on the left of the arrow) and the coverage obtained after using optimization (on the right of the arrow). Yellow regions have a smaller coverage after using optimization, green regions have a better coverage.

Figure 7: Variation of coverage from the current to the proposed solution

\begin{tabular}{|c|c|c|c|c|c|}
\hline 0 & & $116 \% \rightarrow 11 \%$ & $18 \% \rightarrow 121 \%$ & & $292 \% \rightarrow 62 \%$ \\
\hline $41 \% \rightarrow 44 \%$ & $15 \% \rightarrow 26 \%$ & $61 \% \rightarrow 11 \%$ & $2 \% \rightarrow 10 \%$ & $136 \% \rightarrow 28 \%$ & $76 \% \rightarrow 18 \%$ \\
\hline $46 \% \rightarrow 114 \%$ & - & $60 \% \rightarrow 10 \%$ & o & 0 & 0 \\
\hline $3 \% \rightarrow 10 \%$ & & & $62 \% \rightarrow 77 \%$ & $66 \% \rightarrow 15 \%$ & $1435 \% \rightarrow 76 \%$ \\
\hline $59 \% \rightarrow 151 \%$ & 0 & $29 \% \rightarrow 15 \%$ & $20 \% \rightarrow 16 \%$ & $53 \% \rightarrow 21 \%$ & $263 \% \rightarrow 49 \%$ \\
\hline & $8 \% \rightarrow 10 \%$ & & $30 \% \rightarrow 27 \%$ & & \\
\hline
\end{tabular}

Figure 8 represents the distribution of the minimum coverage values for all regions when comparing the current solution with the proposed solution. More precisely, we define ranges of minimum percentage coverages on the x-axis. The y-axis specifies the number of regions with a minimum percentage coverage falling within that range. Orange and grey bars represent this information for the current network and the proposed solution, respectively. The minimum coverage value is higher under the proposed solution than the current solution, but also the average coverage value is higher. Moreover, the values of coverage are more equal between regions, increasing the equity of 
the allocation across the country.

Figure 8: Distribution of coverage values.

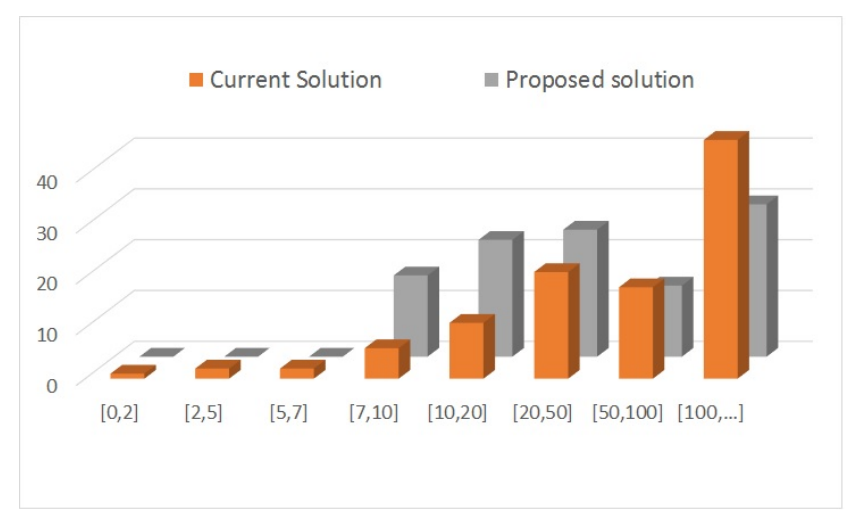

Figure 9: Allocation of supplies by macroarea

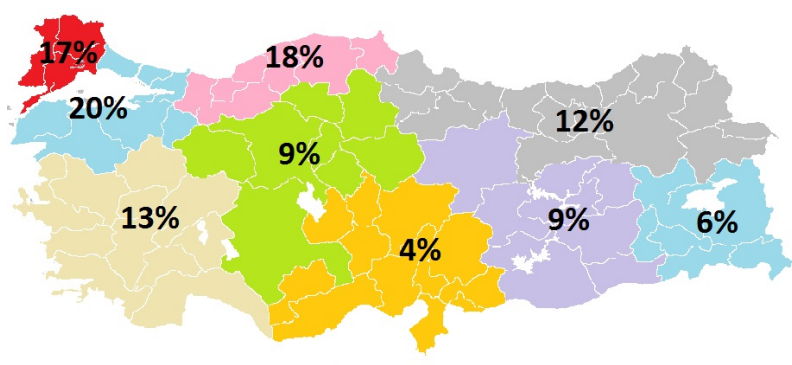

TRC Current

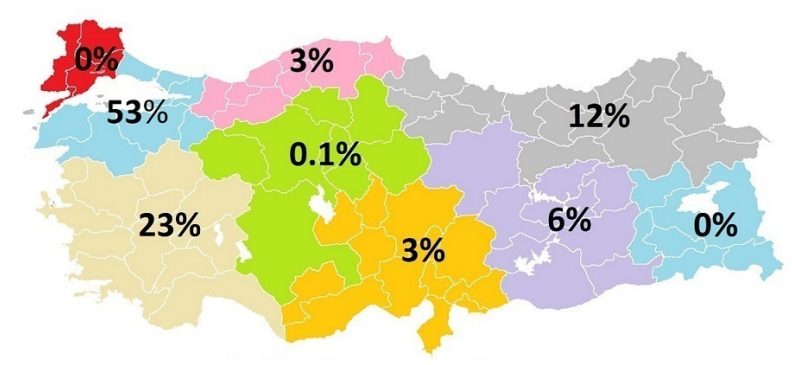

TRC Proposed

Finally, we compare the current and proposed solutions in terms of the quantity of supplies allocated to the warehouses. For ease of representation, we group the supplies stocked in the warehouses in each macroarea together. The resulting map of allocation of supplies is provided in Figure 9. There are similarities between different allocation solutions; however, our method recommends shifting more supplies to the high risk areas. For example, our approach suggests stocking more supplies around Istanbul, Bursa and Izmir (West coast of the country), which represent highly populated and risky areas. Moreover, a lower quantity of supplies are stored in the areas with low risk but high population (i.e., the central region including the capital Ankara). 


\section{Conclusions}

There is a growing body of literature that focuses on developing optimization models to support disaster preparedness decisions. In these models, it is important to effectively characterize and represent uncertainties regarding disaster occurrences and impacts. Most of the existing studies use probabilistic scenarios to represent these uncertainties. In this paper, we propose an alternative practical approach to assess earthquake risk and estimate the size of the affected population in any given region. Our approach adopts an existing earthquake forecasting method from the earthquake engineering literature; specifically, we utilize the risk assessment method developed by Campbell (1982), which relies on statistical analyses of historical earthquake data, and calculate a protected intensity value for each earthquake-prone region. We validate our approach by comparing the computed protected intensity values with the seismic hazard maps of two earthquake-prone countries, namely Turkey and China, which show that our method can assess risk effectively. As discussed by previous studies (e.g., Papoulia et al. (2001)), the effectiveness of Campbell (1982)'s method may be affected by how much historical data is available and by the calibration of the model's parameters. We demonstrate how our method can be successfully attuned to either Turkey or China.

Another advantage of the proposed risk assessment method is that the protected intensity values can be used to directly estimate the number of affected people by using the method of Samardjieva and Badal (2002). As a result, based on historical seismic data, the demand for emergency supplies for any region can be estimated by following a systematic and practical approach, developed based on the existing earthquake engineering literature. We then use the estimated demands to solve the inventory allocation problem faced by the TRC.

The TRC currently allocates supplies in its network primarily by considering the population of the regions, and without using any optimization method. Given a fixed set of warehouses, an inventory allocation model can be applied to better allocate the available stock among the warehouses. We compare the results obtained by our proposed approach and the current solution of the TRC and show that the minimum coverage percentage across all regions significantly increases. Moreover, we show that use of our method produces an allocation of supplies close to highly populated and risky areas. Another suggestion is to stock supplies in a smaller number of warehouses in the network. However, the TRC might be spreading the stocks among a larger number of warehouses to account for the risks associated with losing supplies or difficult accessibility conditions which may appear after the disaster.

In this study, we use a max-min coverage objective which aims to improve the condition of the least advantaged group; min-max/max-min type objectives are frequently used within the context 
of facility location and resource allocation problems to model equity. As discussed in the paper, there can be other ways to make equitable resource allocation decisions in humanitarian settings. In particular, the impact of equity on other metrics as efficiency and effectiveness has been an important concern in studies addressing emergency/disaster settings (e.g., Felder and Brinkmann (2002); Campbell et al. (2008); McCoy and Lee (2014); Ares et al. (2012); Huang et al. (2012)). Developing models which ensure equity without compromising other important metrics is an active research topic relevant to both disaster preparedness and response problems. Therefore, it would be useful to evaluate the implications of alternative objective functions in inventory allocation decisions before presenting an operational plan to the relief organization.

While we illustrate our approach on an inventory allocation problem motivated by a real setting, the proposed technique can be used in other preparedness decisions that need an assessment of earthquake risk. Our approach, which avoids scenarios to represent disaster occurrences and demand for emergency supplies, may also allow for other types of uncertainties in humanitarian operations to be modelled more effectively. Uncertainties related to post-disaster funding, procurement options and transportation/facility disruptions can be isolated from uncertainties regarding earthquake occurrences.

Finally, this paper takes a first step in creating a bridge between the earthquake engineering literature and the OR techniques, used in the context of disaster management. Future research can focus on further interdisciplinary work that adopts alternative techniques from earthquake engineering to improve decision making for disaster preparedness.

\section{Acknowledgments}

We would like to thank Pinar Ozyurt, Sinan Derindere, Kadir Bulut, and Ozge Dogan Irmak from the TRC for providing us with valuable information and data about the TRC supply network and operations. We would like also to thank Prof. Atilla Ansal for his valuable comments and suggestions on forecasting models from earthquake engineering. 


\section{Appendix A. Notation table}

\begin{tabular}{|c|c|}
\hline \multicolumn{2}{|c|}{ Protected earthquake intensity estimation: } \\
\hline$t$ & time horizon for forecasting an earthquake \\
\hline$M_{\max }$ & intensity of the strongest earthquake that occurred in $t$ \\
\hline$m$ & earthquake intensity \\
\hline$m_{l}$ & intensity threshold above which an earthquake is considered a disaster \\
\hline$\nu$ & occurrence rate of earthquake \\
\hline$\beta$ & earthquake intensity rate parameter \\
\hline$A, B, N$ & Gutenberg-Richter relationship terms \\
\hline$n$ & number of earthquakes expected to occur in $t$ \\
\hline$n^{\prime \prime}, m^{\prime \prime}, t^{\prime \prime}, K_{1}, K_{2}$ & $\begin{array}{l}\text { posterior parameters resulting from Bayesian inference on historical earth- } \\
\text { quake data ( } t^{\prime \prime} \text { length of time for which historic data is available, } n^{\prime \prime} \\
\text { number of earthquakes with intensity greater than } m_{l} \text { recorded in } t^{\prime \prime} \text {, and } \\
m^{\prime \prime}=\sum_{i=1}^{n^{\prime \prime}}\left(m_{i}-m_{l}\right) \text { where } m_{i} \text { is the intensity of each recorded earth- } \\
\text { quake, } K_{1} \text { and } K_{2} \text { are normalising parameters) }\end{array}$ \\
\hline$I_{\nu}, I_{\beta}$ & intervals in which the nominal $\nu$ and $\beta$ are expected to lie \\
\hline$E_{\nu}, E_{\beta}$ & expected values of $\nu$ and $\beta$ distributions \\
\hline$\%_{D e v} E_{\nu}, \%_{D e v} E_{\beta}$ & $\begin{array}{l}\text { maximum expected deviation between the expected value of } \nu \text { and } \beta \text { and } \\
\text { their nominal values }\end{array}$ \\
\hline $\bar{\nu}, \underline{\beta}$ & worst case values of $\nu$ and $\beta$ in $I_{\nu}, I_{\beta}$ \\
\hline$\alpha$ & parameter determining the probability of an earthquake occurrence \\
\hline$M_{\alpha, \bar{\nu}, \underline{\beta}}$ & protected earthquake intensity \\
\hline \multicolumn{2}{|c|}{ Number of injured and casualties estimation: } \\
\hline$P$ & population density \\
\hline$a(P), b(P)$ & parameters in the model of Samardjieva and Badal (2002) \\
\hline$N_{c}$ and $N_{i}$ & number of casualties and injured people \\
\hline$R$ & radial distance from epicentre of an earthquake \\
\hline$A_{F}, A_{E}$ & areas of circular and ring-shaped regions around the epicentre \\
\hline \multicolumn{2}{|c|}{ TRC inventory allocation model: } \\
\hline $\mathcal{J}$ & set of TRC facilities \\
\hline $\mathcal{I}$ & set of regions where an earthquake may occur \\
\hline$C_{j}$ & capacity of facility $j \in \mathcal{J}$ \\
\hline$B$ & number of available kits \\
\hline$a_{i j}$ & coefficient of coverage of facility $j \in \mathcal{J}$ to region $i \in \mathcal{I}$ \\
\hline$z$ & minimum coverage level in the country \\
\hline$x_{j}$ & kits allocated to facility $j \in \mathcal{J}$ \\
\hline$D_{i}$ & demand of supplies in region $i \in \mathcal{I}$ \\
\hline $\mathcal{M}$ & set of macroareas \\
\hline$M_{k}$ & set of TRC facilities in the macroarea $k \in \mathcal{M}$ \\
\hline$T_{k}$ & target of kits for macroarea $k \in \mathcal{M}$ \\
\hline
\end{tabular}




\section{Appendix B. China risk assessment example}

The historical data for China is obtained from the National Center for Environmental Information (2015), using the extreme coordinates for the country (as done for Turkey). The same number of rectangles was considered (36 rectangles). The data for this country presents a wider variability, and fewer events were recorded. There are regions with no strong earthquakes in the past 115 years, as well as areas with up to 42 disastrous occurrences on the same time horizon. The strongest earthquake showed an intensity of 8.6 on the Richter scale.

Regions with heterogeneous risk profiles are challenging for risk assessment models, and finding a unique set of $\alpha, t$ and $\%$ Dev values for all regions can be problematic. By setting $\%$ Dev $=0$ (i.e., using expected intensity values and not protected values), we could not find any combination of the values of $\alpha$ and $t$ that would invariably produce suitable intensities. We obtained either regions associated with very small protected intensities (even negative intensities when $t$ is small and the probability of occurrence of an earthquake high) or too large (above 10 Richter scale, with large $t$ and low probability of occurrence of an earthquake). Such variability would not allow the integration of expected intensities with the demand estimation model of Samardjieva and Badal (2002).

The $\%$ Dev value was kept to $5 \%$ in estimating $\beta$, but we increased this value to $\%$ Dev $=47.5$ in estimating $\bar{\nu} . \alpha=0.85$ modelled a lower forecasted probability of occurrence of an earthquake, motivated by the fact that the earthquakes in the data set were roughly one third of those for Turkey. By increasing the $\%$ Dev value associated with the parameter $\nu$ and decreasing $\alpha$, we could obtain a suitable range of protected intensity values. This allowed to have $M_{\alpha, \bar{\nu}, \underline{\beta}}$ values varying between 5 to 9.3 intensity (with an average intensity of 6.6) across the country. A graphical representation is provided in Figure B.10, where our risk assessment is compared with the hazard map provided by the Global Seismic Hazard Assessment Program GSHAP, 2015. Our map shows a good match with the hazard map. 
Figure B.10: Protected intensities and hazard maps, China

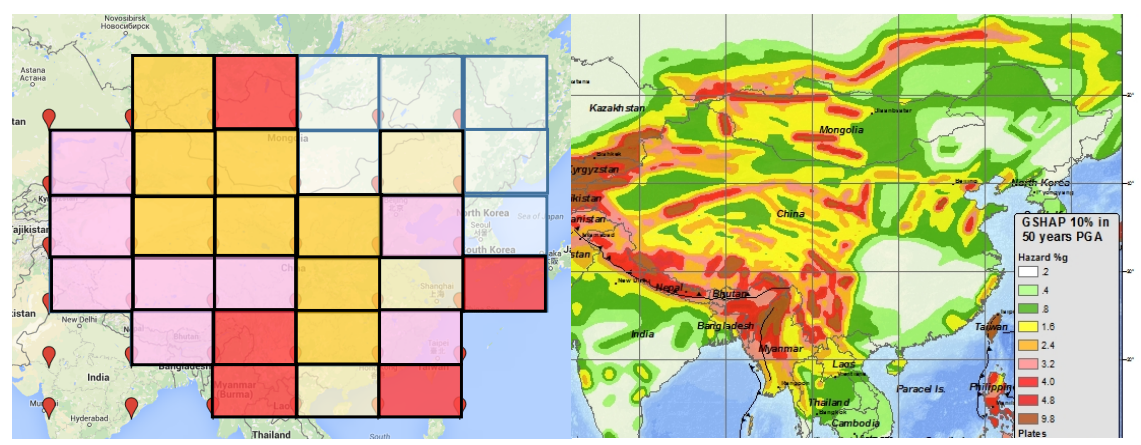

\section{References}

N. Altay and W. G. Green. OR/MS research in disaster operations management. European Journal of Operational Research, 175 (1):475-493, 2006.

E. E. Alvarez. Estimation in stationary Markov renewal processes, with application to earthquake forecasting in Turkey. Methodology and Computing in Applied Probability, 7:119-130, 2005.

T. Anagnos and A. S. Kiremidjian. A review of earthquake occurrence models for seismic hazard analysis. Probabilistic Engineering Mechanics, 3:3-11, 1988.

J. N. Ares, H. De Vries, and D. Huisman. A column generation approach for locating roadside clinics in africa based on effectiveness and equity. European Journal of Operational Research, 254: 1002-1016, 2012.

J. W. Baker. An introduction to probabilistic seismic hazard analysis (PSHA). Technical report, 2008.

B. Balcik and B. Beamon. Facility location in humanitarian relief. International Journal of Logistics: Research and Applications, 11:101-121, 2008.

B. Balcik, B. M. Beamon, and S. Smilowitz. Last mile distribution in humanitarian relief. Journal of Intelligent Transportation System, 12:51-63, 2008.

B. Balcik, C. D. C. Bozkir, and E. Kundakcioglu. A literature review on inventory management in humanitarian supply chains. Surveys in Operations Research and Management Science, Special Issue in Humanitarian Operations, 21:101-116, 2016. 
Y. Bayrak and T. Türker. The determination of earthquake hazard parameters deduced from Bayesian approach for different seismic source regions of Western Anatolia. Pure and Applied Geophysics, 173:205-220, 2016.

J. R. Benjamin. Probabilistic models for seismic force design. Journal of the Structural Division, 94: 1175-1196, 1968.

A. M. Campbell, D. Vandenbussche, and W. Hermann. Models, solutions and enabling technologies in humanitarian logistics. Transportation Science, 42:127-145, 2008.

K. W. Campbell. Bayesian analysis of extreme earthquake occurrences. Bulletin of the Seismological Society of America, 72:1689-1705, 1982.

K. W. Campbell. Bayesian analysis of extreme earthquake occurrences. Part II. application to the San Jacinto fault zone of Southern California. Bulletin of the Seismological Society of America, 73:1099-1115, 1983.

A. M. Caunhye, X. Nie, and S. Pokharel. Optimization models in emergency logistics: A literature review. Socio-Economic Planning Sciences, 46:4-13, 2012. Special Issue: Disaster Planning and Logistics: Part 1.

L. Christoskov, E. Samardjieva, and D. Solakov. Improvement of the approach in determining the possible human losses during strong earthquakes. Bulgarian Geophysical Journal, 4:85-92, 1990.

C. A. Davis. Loss functions for temporal and spatial optimizing of earthquake prediction and disaster preparedness. Pure and Applied Geophysics, 169:1989-2010, 2012.

S. Duran, M. A. Gutierrez, and P. Keskinocak. Pre-positioning of emergency items for CARE International. Interfaces, 41:223-237, 2011.

B. Epstein and C. Lomnitz. A model for the occurrence of large earthquakes. Nature, 211:954-956, 1966.

M. Erdik, V. Doyuran, N. Akkas, and P. Gulkan. A probabilistic assessment of the seismic hazard in Turkey. Tectonophysics, 117:295-344, 1985.

M. Erdik, K. Şeşetyan, M. B. Demircioğlu, C. Zülfikar, U. Hancılar, C. Tüzün, and E. Harmandar. Rapid earthquake loss assessment after damaging earthquakes. In A. Ansal, editor, Perspectives on European Earthquake Engineering and Seismology, volume 34 of Geotechnical, Geological and Earthquake Engineering, pages 53-95. Springer International Publishing, 2014. 
European Commission. Action plan on the Sendai framework for disaster risk reduction 2015-2030. Brussels, 17.6.2016, 2016).

European Geosciences Union. Natural hazards. http://www.egu.eu/policy/science/ natural-hazards/; accessed April 2017, 2017).

S. Felder and H. Brinkmann. Spatial allocation of emergency medical services: minimising the death rate or providing equal access? Regional Science and Urban Economics, 32:27-45, 2002.

O. C. Galanis, T.M. Tsapanos, G.A. Papadopoulos, and A. A. Kiratzi. Bayesian extreme values distribution for seismicity parameters assessment in South America. Journal of the Balkan Geophysical Society, 5:77-86, 2002.

G. Galindo and R. Batta. Prepositioning of supplies in preparation for a hurricane under potential destruction of prepositioned supplies. Socio-Economic Planning Sciences, 47:20-37, 2013.

R. A. Garrido, P. Lamas, and F. J. Pino. A stochastic programming approach for floods emergency logistics. Transportation Research Part E: Logistics and Transportation Review, 75:18-31, 2015.

L. Geggel and S. Writer. Calm before the quake? turkey may be due for the big one, 2014. URL http://www.livescience.com/47827-turkey-seismic-gap.html.

N. Gormez, M. Kksalan, and F. S. Salman. Locating disaster response facilities in istanbul. Journal of the Operational Research Society, 62 (7):1239-1252, 2011.

E. Grass and F. Kathrin. Two-stage stochastic programming in disaster management: A literature survey. Surveys in Operations Research and Management Science, page forthcoming, 2016.

J. Greenhough and I. G. Main. A Poisson model for earthquake frequency uncertainties in seismic hazard analysis. Geophysical Research Letters, 35:1-4, 2008.

GSHAP. Global seismic hazard assessment program. http://www.koeri.boun.edu.tr/sismo/ zeqdb/indexeng. asp (accessed July 2015), 2015.

E. J. Gumbel. Statistics of extremes. Columbia Univ. press, New York, 1958.

M. Huang, K. Smilowitz, and B. Balcik. Models for relief routing: Equity, efficiency and efficacy. Transportation research part E: Logistics and Transportation Review, 48:2-18, 2012.

K. Jaiswal, D. J. Wald, and M. Hearne. Estimating casualties for large earthquakes worldwide using an empirical approach. Technical Report 2009-1136, US Geological Survey, 2009. 
K. Kayabali and M. Akin. Seismic hazard map of Turkey using the deterministic approach. Engineering Geology, 69:127-137, 2003.

KOERI. Bogazici University Kandilli Observatory and Earthquake Research Institute. http://www . seismo.ethz.ch/static/GSHAP/eu-af-me/ (accessed July 2015), 2015.

A. Leiras, I. de Brito Jr., E. Q. Peres, T. R. Bertazzo, and H. T. Y. Yoshizaki. Literature review of humanitarian logistics research: trends and challenges. Journal of Humanitarian Logistics and Supply Chain Management, 4:95-130, 2014.

J. H. McCoy and H. L. Lee. Using fairness models to improve equity in health delivery fleet managemen. Production and Operations Management, 23:965-977, 2014.

R. Mohammadi, S. M. T. F. Ghomi, and F. Jolai. Pre-positioning emergency supplies for earthquake response: A new multi-objective particle swarm optimization algorithm. Applied Mathematical Modelling, 40:5183-5199, 2016.

National Center for Environmental Information. http://www.ngdc.noaa.gov/nndc/struts/form? $\mathrm{t}=101650 \& \mathrm{~s}=1 \& \mathrm{~d}=1$ (accessed July 2015), 2015.

N. Noyan, B. Balcik, and S. Atakan. A stochastic optimization model for designing last mile relief networks. Transportation Science, 50:1092-1113, 2015.

L. Özdamar and M. A. Ertem. Models, solutions and enabling technologies in humanitarian logistics. European Journal of Operational Research, 244:55-65, 2015.

J. Papoulia, G. Stavrakakis, and D. Papanikolaou. Bayesian estimation of strong earthquakes in the inner Messiniakos fault zone, southern Greece, based on seismological and geological data. Journal of Seismology, 5:233-242, 2001.

I. A. Parvez. On the bayesian analysis of the earthquake hazard in the north-east indian peninsula. Natural Hazards, 40:397412, 2007.

N. Perez-Rodriguez and J. Holguin-Veras. Inventory-allocation distribution models for postdisaster humanitarian logistics with explicit consideration of deprivation costs. Transportation Science, 50: 1261-1285, 2015.

C. G. Rawls and M. A. Turnquist. Pre-positioning of emergency supplies for disaster response. Transportation Research Part B: Methodological, 44:521-534, 2010. 
C. F. Richter. Elementary Seismology. W. H. Freeman and Co., San Francisco, 1958.

E. Samardjieva and J. Badal. Estimation of the expected number of casualties caused by strong earthquakes. Bulletin of the Seismological Society of America, 92:2310-2322, 2002.

SEDAC. Socioeconomic Data and Applications Center. http://sedac.ciesin.columbia.edu/ data/set/gpw-v3-population-density-future-estimates (accessed June 2015), 2015.

T. Seher and I. G. Main. A statistical evaluation of a stress-forecast earthquake. Geophysical Journal International, 157:187-193, 2004.

E. So and R. Spence. Estimating shaking-induced casualties and building damage for global earthquake events: a proposed modelling approach. Bulletin of Earthquake Engineering, 11:347-363, 2013.

G. N. Stavrakakis and J. Drakopoulos. Bayesian probabilities of earthquake occurrences in Greece and surrounding areas. Pure and Applied Geophysics, 144:307-319, 1995.

S. Taskin and E. J. Lodree. A Bayesian decision model with hurricane forecast updates for emergency supplies inventory management. Journal of the Operational Research Society, 62:1098-1108, 2011.

P. A. Trunick. Special report: delivering relief to tsunami victims. Logistics Today, 2005.

S. Ünal, S. Çelebioğlu, and B. Özmen. Seismic hazard assessment of Turkey by statistical approaches. Turkish Journal of Earth Sciences, 23:350-360, 2014.

United Nations Office for Disaster Risk Reduction. Global assessment report on disaster risk reduction 2015. making development sustainable: The future of disaster risk management. Geneva, 2015).

L. N. Van Wassenhove. Humanitarian aid logistics: supply chain management in high gear. Journal of the Operational research Society, 57:475-489, 2006.

WIKI. Wikipedia page, Turkey. https://en.wikipedia.org/wiki/Turkey (accessed July 2015), 2015 .

R. Yarar, O. Ergunay, M. Erdik, and N. Eren. Attenuation of intensities in Turkey. In Proceedings of the eight world conference on earthquake engineering, volume 2, pages 343-350, San Francisco, California, 1984. World Conferences on Earthquake Engineering.

A. Yazdani and M. Kowsari. Bayesian estimation of seismic hazards in Iran. Scientia Iranica, 20: 422-430, 2013. 